\title{
OPEN The role of c-Met and VEGFR2 in glioblastoma resistance to bevacizumab
}

\author{
Bruno Carvalho ${ }^{1,2,5,6 \bowtie}$, José Manuel Lopes ${ }^{1,3,5,6}$, Roberto Silva ${ }^{1,3}$, Joana Peixoto ${ }^{1,5,6}$, \\ Dina Leitão ${ }^{1}$, Paula Soares ${ }^{1,5,6}$, Ana Catarina Fernandes ${ }^{4}$, Paulo Linhares ${ }^{1,2,7}$, Rui Vaz ${ }^{1,2,7}$ \& \\ Jorge Lima ${ }^{1,5,6}$
}

Dismal prognosis of glioblastoma (GBM) prompts for the identification of response predictors and therapeutic resistance mechanisms of current therapies. The authors investigated the impact of c-Met, HGF, VEGFR2 expression and microvessel density (MVD) in GBM patients submitted to second-line chemotherapy with bevacizumab. Immunohistochemical expression of c-Met, HGF, VEGFR2, and MVD was assessed in tumor specimens of GBM patients treated with bevacizumab, after progression under temozolomide. Survival analysis was evaluated according to the expression of the aforementioned biomarkers. c-Met overexpression was associated with a time-to-progression (TTP) after bevacizumab of 3 months $(95 \% \mathrm{Cl}, 1.5-4.5)$ compared with a TTP of 7 months $(95 \% \mathrm{Cl}, 4.6-9.4)$ in patients with low or no expression of $c-M e t(p=0.05)$. VEGFR2 expression was associated with a TTP after bevacizumab of 3 months $(95 \% \mathrm{Cl}, 1.8-4.2)$ compared with a TTP of 7 months $(95 \% \mathrm{Cl}, 5.7-8.3)$ in patients with no tumoral expression of VEGFR2 $(p=0.009)$. Concomitant c-Met/VEGFR2 overexpression was associated with worse overall survival (13 months) compared with concomitant c-Met/VEGFR2 negative expression (19 months; $p=0.025$ ). Our data support the hypothesis that c-Met and VEGFR2 overexpression have a role in the development of glioblastoma early resistance and might predict poorer responses to anti-angiogenic therapies.

Despite all therapeutic and research efforts, glioblastoma (GBM) prognosis remains ominous with a median overall survival (OS) of 14.6 months and a 5 -year survival rate of $9.8 \%$ in the temozolomide era ${ }^{1}$. Although median OS has improved to 20.9 months with the recent addition of alternating electric field therapy ${ }^{2}$, progression is inexorable and treatment options for recurrency are scarce, with second-line chemotherapy showing only modest anti-tumoral activity ${ }^{3}$.

Bevacizumab is a recombinant human monoclonal antibody that selectively neutralizes VEGF, which showed improved progression-free survival (PFS) and objective response rates, leading to the US Food and Drug Administration approval for recurrent GBM in $2009^{4,5}$. Although subsequent antiangiogenic phase III clinical trials did not validate the results concerning OS in newly diagnosed and recurrent $\mathrm{GBM}^{6-8}$, bevacizumab remains a cornerstone therapy in the treatment of recurrent GBM, providing symptom alleviation, increased PFS and probably a valid survival benefit, in a subgroup of patients ${ }^{9-12}$.

Defining populations of patients who can benefit most from a particular therapy will improve cost-effectiveness and therapeutic outcomes, avoiding futile or harmful treatments ${ }^{13}$. In this perspective, it is crucial to identify response predictors and therapeutic resistance mechanisms of presently available therapies in GBM patients. There are emerging systemic, circulating, tissue and imaging biological markers, but no validated biomarkers for appropriately selecting GBM patients who might respond to antiangiogenic therapy. While survival benefit from bevacizumab-containing regimens is modest, some patients respond better than others and it is crucial to establish predictive and therapy monitoring markers $^{14}$.

${ }^{1}$ Faculty of Medicine, University of Porto, Alameda Prof. Hernâni Monteiro, 4200-319 Porto, Portugal. ${ }^{2}$ Department of Neurosurgery, Centro Hospitalar Universitário S. João, Alameda Prof. Hernâni Monteiro, 4200-319 Porto, Portugal. ${ }^{3}$ Department of Pathology, Centro Hospitalar Universitário S. João, Alameda Prof. Hernâni Monteiro, 4200-319 Porto, Portugal. " Department of Oncology, Centro Hospitalar Universitário S. João, Alameda Prof. Hernâni Monteiro, 4200-319 Porto, Portugal. ${ }^{5}$ Instituto de Investigação E Inovação Em Saúde (i3S), Porto, Portugal. 'Institute of Molecular Pathology and Immunology, University of Porto (Ipatimup), Porto, Portugal. ${ }^{7}$ Neurosciences Center-CUF Hospital, Estrada da Circunvalação 14341, 4100-180 Porto, Portugal. ${ }^{\square}$ email: bmfcarvalho@gmail.com 
Resistance to antiangiogenic therapies is partly due to a substantial redundancy in proangiogenic ligands and receptors. Evidence suggests that, in the early phase, tumors are dependent on VEGF and resistance emerges from the upregulation of $\mathrm{HGF} / \mathrm{c}-\mathrm{MET}$ pathway ${ }^{15,16}$. In this standpoint, our purpose was to evaluate the prognostic and therapeutic significance of c-Met, HGF and VEGFR2 expression, together with microvessel density (MVD), in patients with recurrent GBM undergoing second-line chemotherapy with bevacizumab-based therapy.

\section{Methods}

Patient and tissue collection. We conducted a retrospective study of 40 consecutive patients with recurrent GBM treated at Centro Hospitalar Universitário São João, Oporto, between January 2008 and October 2013. All patients were treated with radio-chemotherapy according to the Stupp protocol, followed by treatment with bevacizumab-based therapy, upon recurrence.

The inclusion criteria were (i) patients with histologically proven GBM, age $\geq 18$ years; (ii) recurrence after magnetic resonance imaging response assessment; and (iii) second-line treatment with bevacizumab-based regimen after neuro-oncology multidisciplinary team meeting decision.

All patients were treated according to standard first-line chemotherapy with temozolomide, administered $75 \mathrm{mg} / \mathrm{m}^{2}$ concurrent with daily external-beam radiation therapy (RT) (2 Gy/fraction, for a total of $60 \mathrm{~Gy}$ in 30 fractions) and followed by adjuvant TMZ at $150-200 \mathrm{mg} / \mathrm{m}^{2}$ for 5 days every 28 days until progression. All patients were treated with second-line bevacizumab-based therapy, namely bevacizumab $(10 \mathrm{mg} / \mathrm{kg})+$ irinotecan ( 340 or $125 \mathrm{mg} / \mathrm{m}^{2}$, with or without concomitant enzyme inducing antiepileptic drugs, respectively) every two weeks.

Two neuropathologists (JML/RS) retrospectively reviewed the diagnoses after collection of paraffin-embedded tumor specimens. Subsequently, the immunohistochemical (IHC) evaluation of c-Met, HGF, VEGFR2, and MVD (CD31 expression) was performed in representative tumor sections. All cases were IDH-wild type as assessed by Sanger sequencing (IDH1 and $I D H 2)$.

Demographic, clinical, therapeutic and survival data of all patients were collected and analysed. Then, the tissue expression of the aforementioned biomarkers was correlated with survival data, namely OS, time to progression after temozolomide (TTP-1), and time to progression after bevacizumab (TTP-2).

This study was approved by the Local Ethical Committee of Centro Hospitalar de São João (Porto, Portugal) and conducted according to the National Ethical Guidelines.

Immunohistochemistry study. Serial tissue sections ( $3 \mu \mathrm{m}$ thick) of representative tumor samples were prepared on the slide for IHC analysis. Sections were immunostained with the following monoclonal antibodies: c-Met (18-2257, Invitrogen), HGF (H-145:SC-7949 Santa Cruz Biotechnology), VEGFR2 (55B11, Cell Signaling) and CD 31 (JC70 Cell Marque).

For the c-Met IHC, we performed deparaffinization and rehydration of tissue sections, followed by heatinduced antigen retrieval (citrate buffer [pH 6.0] $2 \mathrm{~min}$ at $65^{\circ} \mathrm{C}, 5 \mathrm{~min} 125^{\circ} \mathrm{C}$ in a steamer) and blockage of endogenous peroxidase activity $(0.3 \%$ hydrogen peroxide in methanol). Incubation with the primary antibody against c-Met (dilution 1:100) was conducted overnight at $4{ }^{\circ} \mathrm{C}$, followed by biotinylated secondary antibody, streptavidin-HRP and staining with diaminobenzidine (DAB) chromogen (Dako Carpinteria, CA, USA) and counterstained with haematoxylin. The positive control was a sample of papillary renal carcinoma.

For the HGF IHC, we performed deparaffinization and rehydration of tissue sections, followed by heatinduced antigen retrieval (EDTA buffer [pH 9.0] $5 \mathrm{~min}$ at $98{ }^{\circ} \mathrm{C}$ in a steamer) and blockage of endogenous peroxidase activity $(0.3 \%$ hydrogen peroxide in methanol). Incubation with the primary antibody against HGF (dilution 1:50) was conducted over-night at $4^{\circ} \mathrm{C}$, followed by the same procedure mentioned above. The positive control was a colon sample.

VEGFR2 and CD31 immunostaining was performed using the Ventana Benchmark Ultra automated immunostainer (Ventana Medical System, Tucson, AZ, USA). Briefly, the tissue sections were deparaffinized using EZPrep (VMS, USA) at $72^{\circ} \mathrm{C}$, and washed with Reaction Buffer (VMS, USA). Antigen retrieval was achieved using Cell Conditioning solution 1 (CC1 Universal Buffer Tris/EDTA pH 8.4) (VMS, USA), at a temperature ranging from 95 to $98^{\circ} \mathrm{C}$, followed by blockage of endogenous peroxidase activity using OptiView Peroxidase Inhibitor (3.0\%). Diluent Option (VMS, USA) was also used to decrease unspecific background signal. Primary antibodies were then incubated using the optimized dilutions and time (CD31 antibody 1/100 dilution and 32 min incubation and VEGFR2 antibody $1 / 600$ dilution and $1 \mathrm{~h} 30 \mathrm{~min}$ incubation). The detection of the primary antibody was accomplished using the OptiView Horseradish Peroxidase Universal Multimer (VMS, USA) and the signal revelation was obtained with OptiView cocktail (VMS, USA)-DAB, H2O2 and Copper. Sections were counterstained with Hematoxylin and Bluing Reagent (VMS, USA) and washed in tap water with EZPrep, to remove oil coverslip. Finally, slides were dehydrated using a series of ethanol and xylene and mounted for light microscopy. The specificity of immunohistochemistry was checked using negative and positive controls.

Scoring and interpretation of immunohistochemistry. Sections were examined for c-Met, HGF, VEGFR2 and CD31 immunoreactivity by two neuropathologists blinded to the outcomes and clinical features. Tumors were classified according to the expression intensity and extension, which were then used to reach a composite score (intensity $\times$ extension). For the intensity, tumors were classified as slight or no expressing (grade 1 or 0 , respectively), moderate expression (grade 2 ) or strong expression (grade 3 ). Regarding extension, tumors were classified according to the percentage of positive cells: staining observed in less than $25 \%$ of cells (grade 1), in a sizable subgroup of cells (25-50\%; grade 2$)$, most cells (50-75\%; grade 3 ), or nearly all cells ( $>75 \%$; grade 4 ) in the high-power field. Tumors displaying moderate immunopositivity in $>50 \%$ of tumor cells or strong immunopositivity in $>25 \%$ of tumor cells were considered to be positive tumors; all other cases were considered 


\begin{tabular}{|c|c|}
\hline Variables & N (\%) \\
\hline \multicolumn{2}{|l|}{ Gender } \\
\hline $\begin{array}{l}\text { Male } \\
\text { Female }\end{array}$ & $\begin{array}{l}28(70) \\
12(30)\end{array}$ \\
\hline $\begin{array}{l}\text { Age (years), median } \\
\text { Range }\end{array}$ & $\begin{array}{l}59 \\
42-73\end{array}$ \\
\hline \multicolumn{2}{|l|}{ ECOG } \\
\hline $\begin{array}{l}0 \\
1 \\
2 \\
3\end{array}$ & $\begin{array}{l}21(52.5) \\
15(37.5) \\
2(5.0) \\
2(5.0)\end{array}$ \\
\hline \multicolumn{2}{|l|}{ Focality } \\
\hline $\begin{array}{l}\text { Unifocal } \\
\text { Multifocal }\end{array}$ & $\begin{array}{l}37(92.5) \\
3(7.5)\end{array}$ \\
\hline \multicolumn{2}{|l|}{ Type of surgery } \\
\hline $\begin{array}{l}\text { Total resection } \\
\text { Partial resection } \\
\text { Biopsy }\end{array}$ & $\begin{array}{l}22(55) \\
12(30) \\
6(15)\end{array}$ \\
\hline $\begin{array}{l}\text { Number of TMZ cycles, median } \\
\text { Range }\end{array}$ & $\begin{array}{l}5 \\
1-28\end{array}$ \\
\hline $\begin{array}{l}\text { mOS, months } \\
95 \% \mathrm{CI}\end{array}$ & $\begin{array}{l}18 \\
16.2-19.8\end{array}$ \\
\hline $\begin{array}{l}\text { mTTP-1, months } \\
95 \% \text { CI }\end{array}$ & \begin{tabular}{|l|}
8 \\
$6.8-9.2$
\end{tabular} \\
\hline $\begin{array}{l}\text { mTTP-2, months } \\
95 \% \text { CI }\end{array}$ & \begin{tabular}{|l|}
5 \\
$2.9-7.1$
\end{tabular} \\
\hline
\end{tabular}

Table 1. Clinical and demographic characteristics of GBM patients. ECOG Eastern Cooperative Oncology Group performance status, TMZ temozolomide, $m O S$ median overall survival, $m T T P-1$ median time to progression after temozolomide, $m$ TTP-2 median time to progression after bevacizumab, 95\% CI 95\% confidence interval.

negative. For statistical purposes, immunoreactivity was analyzed as a dichotomous covariate classified as negative or weak versus positive or strongly positive ${ }^{17}$.

For evaluating MVD, the three most vascularized areas (hot spots) were selected in sections stained for CD31, using low-power field $(\times 40)$ magnification. The counting of vessels was performed in these areas, using highpower field $\left(\times 200,0.1 \mathrm{~mm}^{2}\right)$ and avoiding areas of sclerosis, necrosis, and non-neoplastic tissue ${ }^{18,19}$. The number of CD31-positive vessels $/ 0.1 \mathrm{~mm}^{2}$ of tumor tissue was calculated in three hotspots and its average was used to indicate MVD.

Data and statistical analysis. The primary endpoint of this study was to correlate the expression of c-Met, HGF, VEGFR2 and the MVD with time to progression after bevacizumab (TTP-2), and secondarily with time to progression after temozolomide (TTP-1) and OS. Differences between groups were analysed using Chi-square, Fisher's exact test or Mann-Whitney U test as appropriate. Survival data was evaluated using Kaplan-Meier product-limit analysis and 95\% confidence intervals (CI) were calculated. The Log-rank test was used to detect statistically significant differences in survival distribution. Multivariate survival analysis was calculated using the Cox proportional hazard method to settle on independent prognostic factors. Differences were considered statistically significant at $\mathrm{P}<0.05$. The software used for statistical analysis was IBM SPSS Statistics 25.

Ethics approval and consent to participate. This study was approved by the Local Ethical Committee of Centro Hospitalar de São João, (Porto, Portugal)/Medical Faculty (Porto, Portugal) and was conducted according to the National Ethical Guidelines. Informed consent was waived by the Local Ethical Committee of Centro Hospitalar S. João/Faculty of Medicine of the University of Porto (Comissão de Ética para a Saúde do Centro Hospitalar S. João/Faculdade de Medicina da Universidade do Porto). The study was performed in accordance with the Declaration of Helsinki.

\section{Results}

Patients demographics and clinical outcome. The summary of demographic, clinical, therapeutic and survival data of the cohort is presented in Table 1 . The median age at diagnosis was 59 years (42-73). The majority of patients were male $(70 \%, n=28)$ and $90 \%(n=36)$ had an ECOG performance status of 0 or 1 . Complete resection was achieved in 22 patients (55\%), partial resection in 12 patients (30\%) and biopsy in 6 patients (15\%). Median OS was 18 months (95\% CI 16.2-19.8) for the entire cohort. The median time to progression after temozolomide (mTTP-1) was 8 months (95\% CI 6.8-9.2) and the median time to progression after bevacizumab (mTTP-2) was 5 months (95\% CI 2.9-7.1). 

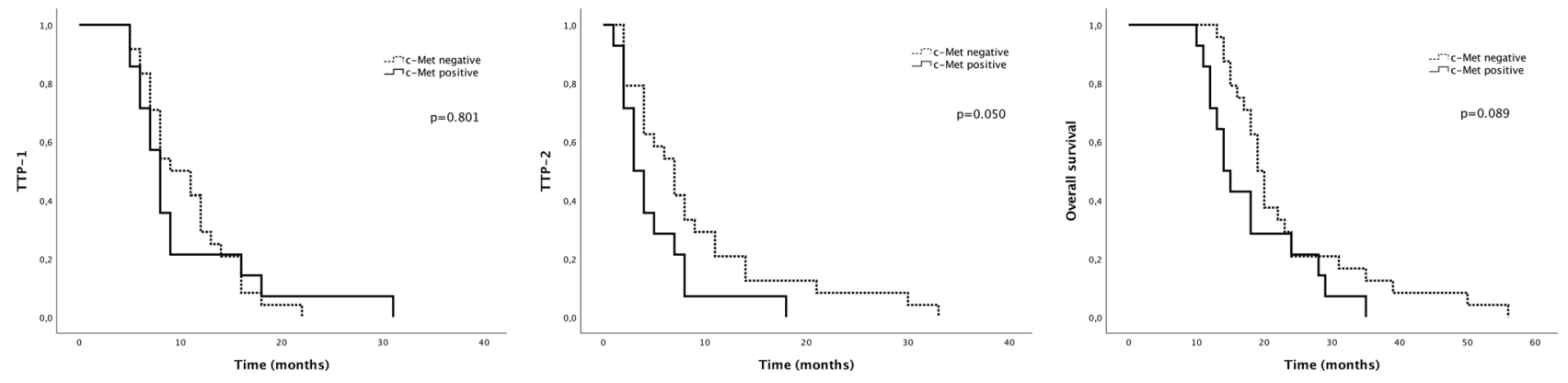

Figure 1. Kaplan-Meier analysis of survival curves according to c-Met expression.
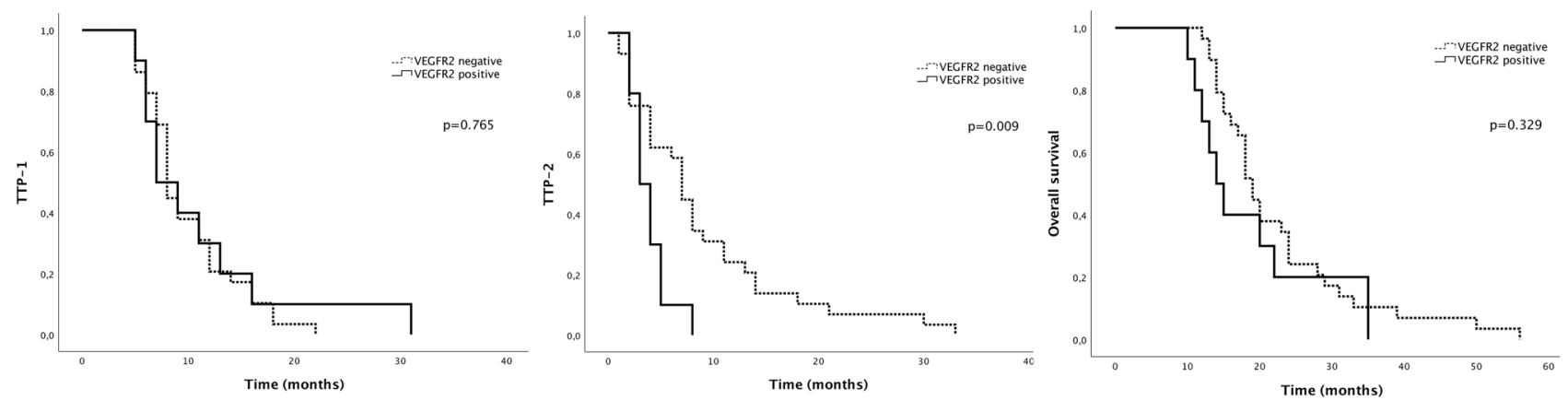

Figure 2. Kaplan-Meier analysis of survival curves according to VEGFR2 expression.

Biomarker expression and survival analysis. The expression of biomarkers was detected as follows: c-Met overexpression was detected in $36.8 \%$ (14 of 38) of the patients, HGF overexpression was detected in $35.3 \%$ ( 12 of 34 ) of the patients and VEGFR2 overexpression was detected in $25.6 \%$ (10 of 39) of the patients.

The expression of c-Met had no impact on TTP-1. Patients with GBM overexpressing c-Met had a mTTP-2 of 3 months (95\% CI 1.5-4.5) versus 7 months (95\% CI 4.6-9.4) in patients with GBM with little or no expression of $\mathrm{c}$-Met expression (Log-rank test, $\mathrm{p}=0.050)$. There was a trend towards better median OS in patients with c-Met negative tumors (19 months, 95\% CI 17.4-20.6) versus patients with c-Met positive tumors (14 months, 95\% CI 11.6-16.5) (Log-rank test, p=0.089) (Fig. 1).

The results for VEGFR2 expression were similar to c-Met: while there was no impact on TTP-1, the TTP-2 of patients with VEGFR2-overexpressing GBM was significantly shorter than the TTP2 of patients with VEGFR2negative GBM (3 months versus 7 months, respectively, $\mathrm{p}=0.009$ ). In VEGFR2-negative group the response rate was $62 \%$ (18 out of 29 patients) versus $10 \%$ ( 1 out of 10 patients) in VEGFR2-positive group (Fisher's exact test, $\mathrm{p}=0.008$ ). There was no difference in median OS between patients with VEGFR2-positive and negative tumors (14 months versus 19 months, respectively, $\mathrm{p}=0.329$ ) (Fig. 2).

Concerning HGF, there were no significant differences between patients with HGF-positive and negative tumors.

The number of CD31-positive vessels $/ 0.1 \mathrm{~mm}^{2}$ of tumor tissue was used to calculate MVD. The median value of MVD was 17.65 (range 2.7-36.3) vessels/ $0.1 \mathrm{~mm}^{2}$. We did not find any statistically significant difference between patients with higher (above median) MVD and lower MVD regarding TTP-1, TTP-2 or OS. High MVD group showed TTP- 1 of 9 months (versus 8 months in low MVD group, $p=0.535$ ), TTP- 2 of 7 months (versus 4 months in low MVD group, $\mathrm{p}=0.297$ ) and an OS of 19 months (versus 16 months in low MVD group, $\mathrm{p}=0.090)$.

Concomitant overexpression of c-Met and VEGFR2 was detected in 18.9\% (7 of 37) of the patients. This concurrent expression had no impact on TTP-1 ( 8 versus 7 months, $\mathrm{p}=0.658$ ), but was significantly associated with worse TTP-2 (3 months) compared with concomitant negative expression of c-Met and VEGFR2 (7 months, $\mathrm{p}=0.001$ ). Concomitant overexpression of $\mathrm{c}$-Met and VEGFR2 was significantly associated with worse OS (13 months) compared with concomitant negative expression of c-Met and VEGFR2 (19 months, $\mathrm{p}=0.025)$ (Fig. 3). Representative images of immunopositivity are depicted in Fig. 4.

A comparative analysis of the clinicopathological features between c-Met positive and negative GBM showed no significant differences concerning age $(p=0.797$, Mann-Whitney $U$ test $)$, gender $(p=0.728$, Fisher's exact test), ECOG status ( $p=0.167$, Mann-Whitney $U$ test), type of surgery ( $p=0.240$, Chi-square test) or the number of temozolomide cycles $(\mathrm{p}=0.148$, Mann-Whitney $\mathrm{U}$ test $)$. The same was observed when comparing HGF and VEGFR2-positive and negative GBM.

Multivariate analysis of covariates on TTP-2 (including age, ECOG, type of surgery, c-Met and VEGFR2) revealed that $c$-Met expression $(p=0.045$, HR 2.31) and VEGFR2 expression $(p=0.039$, HR 2.59) are independent predictors of progression while on bevacizumab. (Table 2) No significant impact on prognosis was observed in OS multivariate analysis. 

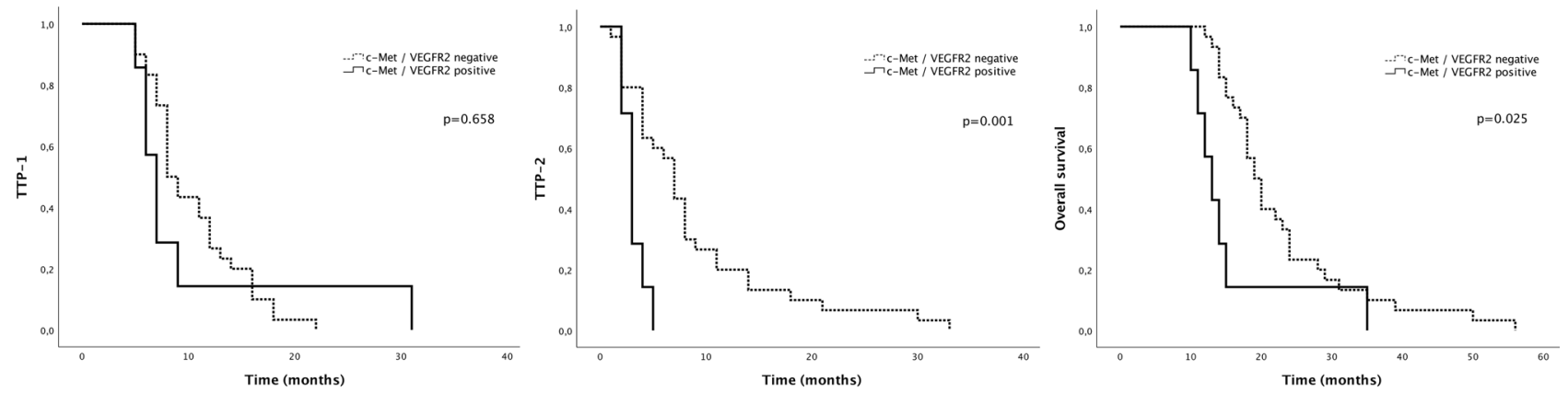

Figure 3. Kaplan-Meier analysis of survival curves according to concomitant c-Met/VEGFR2 expression.
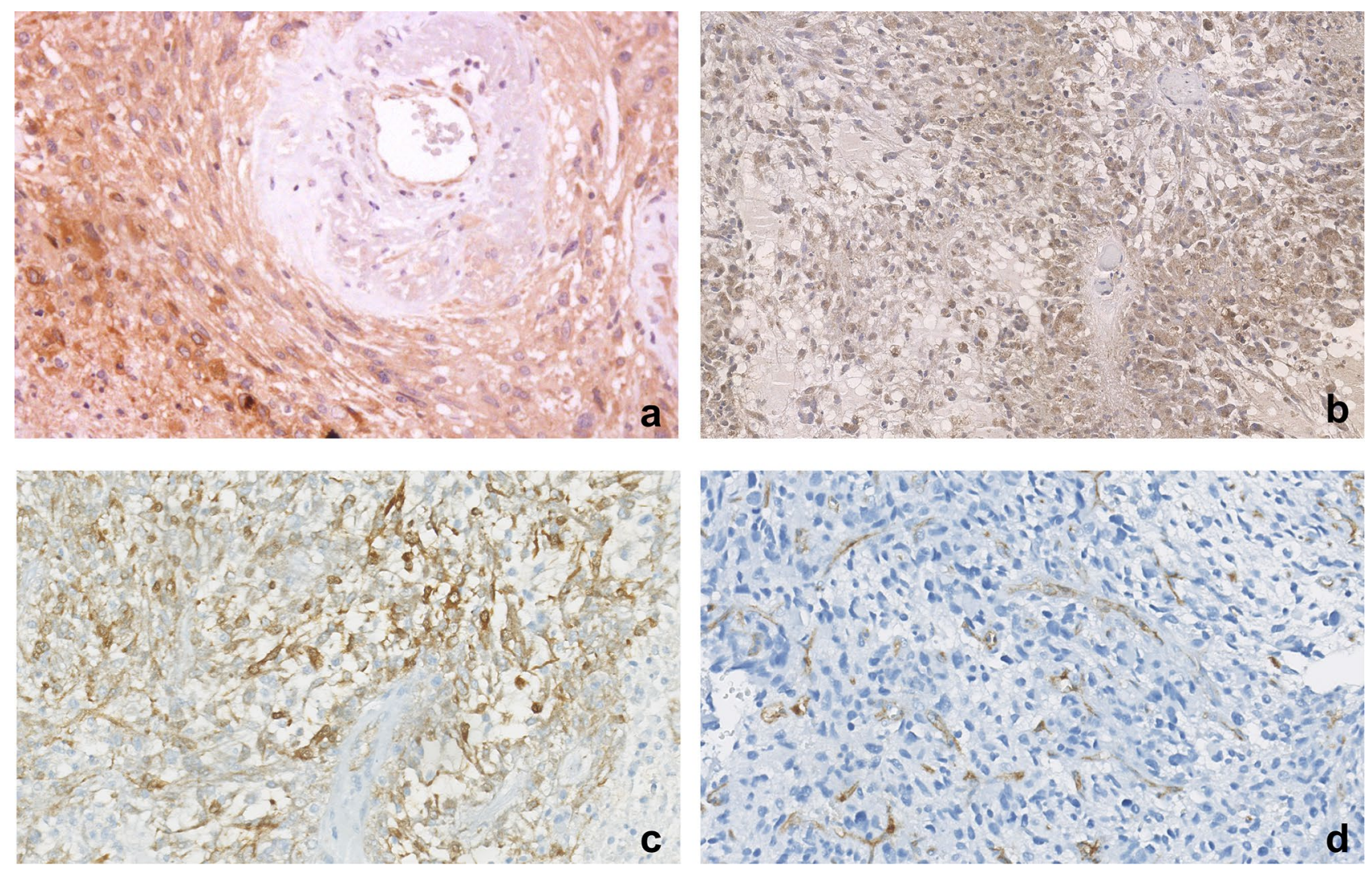

Figure 4. Representative positive immunohistochemical staining with c-Met (a), HGF (b), VEGFR2 (c) and CD31 (d) (original magnification $\times 200)$.

\begin{tabular}{|l|l|l|l|}
\hline \multirow{2}{*}{ Variable } & \multicolumn{3}{|l|}{ Multivariate analysis } \\
\cline { 2 - 4 } Age & HR & $\mathbf{9 5 \%}$ CI & p value \\
\hline$\geq 65$ versus $<65$ years & 1.63 & $0.68-3.93$ & 0.277 \\
\hline ECOG \\
\hline $0-1$ versus $\geq 2$ & 0.271 & $0.07-1.04$ & 0.058 \\
\hline Type of surgery & 1.18 & $0.39-3.50$ & 0.77 \\
\hline c-Met expression & $\mathbf{2 . 3 1}$ & $\mathbf{1 . 0 2 - 5 . 2 3}$ & $\mathbf{0 . 0 4 5}$ \\
\hline VEGFR2 expression & $\mathbf{2 . 5 9}$ & $\mathbf{1 . 0 5 - 6 . 3 9}$ & $\mathbf{0 . 0 3 9}$ \\
\hline
\end{tabular}

Table 2. Analysis of independent predictors of time to progression of GBM patients while on bevacizumab (TTP-2) based-therapy. HR hazard ratio. Results indicated in bold are significant 


\section{Discussion}

Tumor angiogenesis is essential for tumor growth and invasion, relying on a highly complex network of growth factor signaling, microvascular proliferation, extracellular matrix remodeling, and stromal cell interactions. Despite promising preclinical and clinical results, antiangiogenic therapies led only to mild survival benefits. In GBM, randomized clinical trials (RCT) conducted in the primary setting (AVAglio and RTOG 0825) demonstrated benefits in PFS but no impact on OS. Moreover, the clinical prognostic factors, MGMT status and molecular markers did not seem to be predictive of response ${ }^{6,7}$. On the other hand, the BELOB phase 2 trial supported the use of bevacizumab in combination with lomustine in the recurrent GBM setting, showing a benefit in OS; however, it was not validated in EORTC 26101 phase 3 trial. The latter showed PFS benefit but no OS advantage. Notwithstanding these results, it is worth mentioning that crossovers to off-label use of bevacizumab occurred in $35.5 \%$ of the monotherapy group and $18.7 \%$ of the combination group ${ }^{3,8}$.

The minimal survival benefits observed in several studies can be secondary to intrinsic or acquired resistance, through the activation of alternative mechanisms that sustain tumor vascularization and growth ${ }^{15,16}$. These mechanisms of resistance can be subdivided into VEGF-dependent alterations, VEGF-independent pathways and stromal cell interactions. Therefore, complementary and novel approaches, such as the combination of these inhibitors with agents targeting alternative mechanisms of blood vessel formation, are urgently needed in order to overcome resistance and improve outcomes ${ }^{15}$.

To date, no subgroup has been identified that consistently benefits from VEGF inhibition ${ }^{11}$. Several putative clinical factors (hypertension and proteinuria) ${ }^{20}$, tissue markers (VEGF-A, VEGFR2, c-Met) ${ }^{16,21,22}$, circulating biomarkers (MMP2) ${ }^{23}$, imaging perfusion and diffusion maps ${ }^{24}$ and molecular markers (EGFR, Proneural subtype $)^{25-27}$ have been investigated, although they lack validation and confirmation among studies.

Some authors have identified c-Met receptor tyrosine kinase as having a role in resistance mechanisms driving GBM invasion in xenografts. This resistance to antiangiogenic agents may be mediated by the upregulation of c-Met gene expression (acquired resistance) or due to a selective survival of subpopulations of tumor cells that overexpress c-Met (intrinsic resistance) ${ }^{16,28,29}$.

In our study we aimed to evaluate the prognostic and therapeutic significance of proangiogenic ligands and receptors (c-Met, HGF, VEGFR2) and MVD, specifically in a clinical population of bevacizumab-treated recurrent GBM patients. Herewith, we attempt to identify which subgroup of patients has an obvious benefit from anti-angiogenic therapy and which subgroup is mainly resistant to this therapy.

Our study detected c-Met overexpression in $36.8 \%$ of the patients and revealed a significant reduction in TTP after antiangiogenic therapy, while not affecting TTP after temozolomide. In line with existing evidence that report c-Met overexpression in $34 \%$ of $\mathrm{GBM}^{17}$, there was a trend towards a shorter OS time in c-Metoverexpressing tumors. This underscores the role of c-Met in early resistance and tumor invasiveness. VEGFR2 overexpression by tumor cells was detected in $25.6 \%$ of the patients and - similarly to c-Met-was associated only with a shorter TTP after antiangiogenic therapy. Kessler et al. detected VEGFR2 immunopositivity in 19\% of the specimens and showed increased aggressiveness and shorter survival associated with VEGFR2 overexpression in glioma cells, by hampering antiangiogenesis and inducing a proinvasive response to bevacizumab ${ }^{21}$.

Although some authors ${ }^{30}$ have found in vitro and in vivo evidence that HGF co-expression is a key predictor of sensitivity to c-Met inhibitors, in our study HGF expression was not predictive of differences in TTP-1, TTP-2 and OS.

Tumor MVD is reported as a potential predictor of bevacizumab benefit in other malignancies, such as ovarian cancer, showing a positive association between MVD and magnitude of bevacizumab effect ${ }^{31}$. In GBM, Tamura et al. showed that antiangiogenic therapy induces the apparent vascular normalization and that peritumoral immature vessels may be resistant to treatment with VEGFR-targeting drugs ${ }^{32}$. In our study we did not find any association between MVD and TTP or OS. Other markers, such as endoglin, are highly expressed on actively proliferating endothelial cells and may increase specificity in angiogenesis evaluation in $\mathrm{GBM}^{33}$.

Despite some studies reporting significant associations and promising predictive markers, others have not accomplished the same conclusions. Kopp et al. studied proliferation, molecular, vascular, and radiological markers in 33 patients with recurrent GBM treated with bevacizumab in the first recurrence, but did not find any predictive biomarker of response or survival benefit for antiangiogenic therapy ${ }^{34}$.

Remarkably, the worst TTP-2 and OS was found in GBM with concomitant overexpression of c-Met and VEGFR2, which denotes primary/innate activation of both angiogenesis pathways. This data emphasizes the rational of combining angiogenesis inhibition with drugs that target evasive resistance mechanisms to provide more durable efficacy. In this perspective, c-Met upregulation seems to be a putative major factor for antiangiogenic resistance. Therapeutically targeting c-Met and disrupting c-Met/HGF pathway alone or in combination with antiangiogenics may be a strategy to prevent or overcome resistance and enhance therapeutic efficacy.

In vitro and in vivo synergistic effect of anti-MET and VEGF combination therapy for MET-overexpressing GBM has been reported ${ }^{35}$. At this time, several drugs targeting c-Met are available in clinical trials ${ }^{36}$. Selecting the best combination therapy and subgroup of patients may be the key to overcome resistance and invasiveness in GBM. Some recent clinical trials involving onartuzumab ${ }^{37}$ and capmatinib ${ }^{38}$ uncovered no clinical benefit, but demand more stringent molecular selection strategies. RCT analyzes highlight significant heterogeneity regarding co-occurring genetic alterations that are consistently described in glioblastoma landscape. Additionally, both MET copy number and protein overexpression may have predictive value, but appropriate cut-offs need to be established. Also, the extent of brain exposure to MET inhibition is unknown and may affect outcome. Others have suggested that HGF expression or MGMT unmethylated status may confer benefit. Other RCT are currently ongoing, such as NCT02386826, which addresses the combination of INC280 (capmatinib) and bevacizumab.

Our study adds valuable data to the current body of evidence, suggesting that patient stratification according to the expression of angiogenesis-related receptors may predict antiangiogenic treatment efficacy. Indeed, in this 
series of patients, c-Met and VEGFR2 were noticeably associated with differences in survival under bevacizumab therapy, while HGF and MVD did not display any impact.

As limitations of our study we should point out that it is a retrospective study with a limited number of patients, which may have precluded some significant associations. Also, MGMT methylation was not consistently available. Nonetheless, a thorough pathological and IHC evaluation, detailed clinical, therapeutic, and survival data uncovered significant data.

\section{Conclusion}

Our data substantiate the assumption that c-Met and VEGFR2 overexpression might play a role in developing GBM early resistance and are associated with worse response to antiangiogenic therapies. There is a need for additional studies with prospective validation of these putative biomarkers in the diagnostic stratification of recurrent GBM clinical trials.

\section{Data availability}

The datasets generated and/or analyzed during the current study are not publicly available but are available from the corresponding author upon request.

Received: 28 October 2020; Accepted: 24 February 2021

Published online: 16 March 2021

\section{References}

1. Stupp, R. et al. Effects of radiotherapy with concomitant and adjuvant temozolomide versus radiotherapy alone on survival in glioblastoma in a randomised phase III study: 5-year analysis of the EORTC-NCIC trial. Lancet Oncol. 10, 459-466. https://doi. org/10.1016/S1470-2045(09)70025-7 (2009).

2. Stupp, R. et al. Effect of tumor-treating fields plus maintenance temozolomide vs maintenance temozolomide alone on survival in patients with glioblastoma: A randomized clinical trial. JAMA 318, 2306-2316. https://doi.org/10.1001/jama.2017.18718 (2017).

3. Taal, W. et al. Single-agent bevacizumab or lomustine versus a combination of bevacizumab plus lomustine in patients with recurrent glioblastoma (BELOB trial): A randomised controlled phase 2 trial. Lancet Oncol. 15, 943-953. https://doi.org/10.1016/S1470 -2045(14)70314-6 (2014).

4. Cohen, M. H., Shen, Y. L., Keegan, P. \& Pazdur, R. FDA drug approval summary: Bevacizumab (Avastin) as treatment of recurrent glioblastoma multiforme. Oncologist 14, 1131-1138. https://doi.org/10.1634/theoncologist.2009-0121 (2009).

5. Friedman, H. S. et al. Bevacizumab alone and in combination with irinotecan in recurrent glioblastoma. J. Clin. Oncol. 27, 4733-4740. https://doi.org/10.1200/JCO.2008.19.8721 (2009).

6. Chinot, O. L. et al. Bevacizumab plus radiotherapy-temozolomide for newly diagnosed glioblastoma. N. Engl. J. Med. 370, 709-722. https://doi.org/10.1056/NEJMoa1308345 (2014).

7. Gilbert, M. R. et al. A randomized trial of bevacizumab for newly diagnosed glioblastoma. N. Engl. J. Med. 370, 699-708. https:// doi.org/10.1056/NEJMoa1308573 (2014).

8. Wick, W. et al. Lomustine and bevacizumab in progressive glioblastoma. N. Engl. J. Med. 377, 1954-1963. https://doi.org/10.1056/ NEJMoa1707358 (2017).

9. Field, K. M., Jordan, J. T., Wen, P. Y., Rosenthal, M. A. \& Reardon, D. A. Bevacizumab and glioblastoma: Scientific review, newly reported updates, and ongoing controversies. Cancer 121, 997-1007. https://doi.org/10.1002/cncr.28935 (2015).

10. Jubb, A. M. \& Harris, A. L. Biomarkers to predict the clinical efficacy of bevacizumab in cancer. Lancet Oncol. 11, 1172-1183. https ://doi.org/10.1016/S1470-2045(10)70232-1 (2010).

11. Stupp, R. \& Weller, M. Questions regarding the optimal use of bevacizumab in glioblastoma: A moving target. Neuro Oncol. 16, 765-767. https://doi.org/10.1093/neuonc/nou092 (2014).

12. Carvalho, B. F. et al. Second-line chemotherapy in recurrent glioblastoma: A 2-cohort study. Oncol. Res. Treat. 38, 348-354. https ://doi.org/10.1159/000431236 (2015).

13. Mayer, T. M. Can we predict bevacizumab responders in patients with glioblastoma?. J. Clin. Oncol. 33, 2721-2722. https://doi. org/10.1200/JCO.2015.62.3637 (2015).

14. Jain, R. K. et al. Biomarkers of response and resistance to antiangiogenic therapy. Nat. Rev. Clin. Oncol. 6, 327-338. https://doi. org/10.1038/nrclinonc.2009.63 (2009).

15. Haibe, Y. et al. Resistance mechanisms to anti-angiogenic therapies in cancer. Front. Oncol. 10, 221. https://doi.org/10.3389/ fonc.2020.00221 (2020).

16. Jahangiri, A. et al. Gene expression profile identifies tyrosine kinase c-Met as a targetable mediator of antiangiogenic therapy resistance. Clin. Cancer Res. 19, 1773-1783. https://doi.org/10.1158/1078-0432.CCR-12-1281 (2013).

17. Kong, D. S. et al. Prognostic significance of c-Met expression in glioblastomas. Cancer 115, 140-148. https://doi.org/10.1002/ cncr.23972 (2009).

18. Tamura, R. et al. Histopathological investigation of glioblastomas resected under bevacizumab treatment. Oncotarget 7, 5242352435. https://doi.org/10.18632/oncotarget.9387 (2016).

19. Weidner, N., Semple, J. P., Welch, W. R. \& Folkman, J. Tumor angiogenesis and metastasis-Correlation in invasive breast carcinoma. N. Engl. J. Med. 324, 1-8. https://doi.org/10.1056/NEJM199101033240101 (1991).

20. Carvalho, B. et al. Hypertension and proteinuria as clinical biomarkers of response to bevacizumab in glioblastoma patients. J. Neurooncol. 147, 109-116. https://doi.org/10.1007/s11060-020-03404-z (2020).

21. Kessler, T. et al. Glioma cell VEGFR-2 confers resistance to chemotherapeutic and antiangiogenic treatments in PTEN-deficient glioblastoma. Oncotarget 6, 31050-31068. https://doi.org/10.18632/oncotarget.2910 (2015).

22. Nishikawa, R. et al. Biomarker (BM) evaluations in the phase III AVAglio study of bevacizumab (Bv) plus standard radiotherapy (RT) and temozolomide (T) for newly diagnosed gliobastoma (GBM). J. Clin. Oncol. 31, https://doi.org/10.1200/jco.2013.31.15_ suppl.2023 (2013).

23. Tabouret, E. et al. Association of matrix metalloproteinase 2 plasma level with response and survival in patients treated with bevacizumab for recurrent high-grade glioma. Neuro Oncol. 16, 392-399. https://doi.org/10.1093/neuonc/not226 (2014).

24. Petrova, L. et al. Cerebral blood volume and apparent diffusion coefficient-Valuable predictors of non-response to bevacizumab treatment in patients with recurrent glioblastoma. J. Neurol. Sci. 405, 116433. https://doi.org/10.1016/j.jns.2019.116433 (2019).

25. Choi, S. W. et al. Identification of transcriptome signature for predicting clinical response to bevacizumab in recurrent glioblastoma. Cancer Med. 7, 1774-1783. https://doi.org/10.1002/cam4.1439 (2018).

26. Hovinga, K. E. et al. EGFR amplification and classical subtype are associated with a poor response to bevacizumab in recurrent glioblastoma. J. Neurooncol. 142, 337-345. https://doi.org/10.1007/s11060-019-03102-5 (2019). 
27. Sandmann, T. et al. Patients with proneural glioblastoma may derive overall survival benefit from the addition of bevacizumab to first-line radiotherapy and temozolomide: Retrospective analysis of the AVAglio Trial. J. Clin. Oncol. 33, 2735-2744. https://doi. org/10.1200/JCO.2015.61.5005 (2015).

28. McCarty, J. H. Glioblastoma resistance to anti-VEGF therapy: Has the challenge been MET?. Clin. Cancer Res. 19, $1631-1633$. https://doi.org/10.1158/1078-0432.CCR-13-0051 (2013).

29. Lu, K. V. \& Bergers, G. Mechanisms of evasive resistance to anti-VEGF therapy in glioblastoma. CNS Oncol. 2, 49-65. https://doi. org/10.2217/cns.12.36 (2013).

30. Zhang, Y. et al. Hepatocyte growth factor sensitizes brain tumors to c-MET kinase inhibition. Clin. Cancer Res. 19, 1433-1444. https://doi.org/10.1158/1078-0432.CCR-12-2832 (2013).

31. Bais, C. et al. Tumor microvessel density as a potential predictive marker for bevacizumab benefit: GOG-0218 biomarker analyses. J. Natl. Cancer Inst. 109, https://doi.org/10.1093/jnci/djx066 (2017).

32. Tamura, R. et al. Histopathological vascular investigation of the peritumoral brain zone of glioblastomas. J. Neurooncol. 136, 233-241. https://doi.org/10.1007/s11060-017-2648-9 (2018).

33. Nassiri, F. et al. Endoglin (CD105): A review of its role in angiogenesis and tumor diagnosis, progression and therapy. Anticancer Res. 31, 2283-2290 (2011).

34. Manneh Kopp, R. A. et al. Correlation of radiological and immunochemical parameters with clinical outcome in patients with recurrent glioblastoma treated with bevacizumab. Clin. Transl. Oncol. 21, 1413-1423, https://doi.org/10.1007/s12094-019-02070 $-6(2019)$.

35. Okuda, T. et al. Efficacy of combination therapy with MET and VEGF inhibitors for MET-overexpressing glioblastoma. Anticancer Res. 37, 3871-3876. https://doi.org/10.21873/anticanres.11767 (2017).

36. Zhang, Y. et al. Function of the c-Met receptor tyrosine kinase in carcinogenesis and associated therapeutic opportunities. Mol. Cancer 17, 45. https://doi.org/10.1186/s12943-018-0796-y (2018).

37. Cloughesy, T. et al. Randomized, double-blind, placebo-controlled, multicenter phase II study of onartuzumab plus bevacizumab versus placebo plus bevacizumab in patients with recurrent glioblastoma: Efficacy, safety, and hepatocyte growth factor and $\mathrm{O}(6)$ methylguanine-DNA methyltransferase biomarker analyses. J. Clin. Oncol. 35, 343-351. https://doi.org/10.1200/JCO.2015.64.7685 (2017).

38. van den Bent, M. et al. A phase Ib/II, open-label, multicenter study of INC280 (capmatinib) alone and in combination with buparlisib (BKM120) in adult patients with recurrent glioblastoma. J. Neurooncol. 146, 79-89. https://doi.org/10.1007/s11060-019-03337 $-2(2020)$.

\section{Author contributions}

Conceptualization and design of the work: B.C. and J.L. Acquisition and analysis of data: B.C., J.M.L., R.S., J.P., D.L. and A.C.F. Interpretation of data: B.C., J.M.L., R.S., P.L., R.V. and J.L. Original draft preparation: B.C. Review and editing: all authors. Final approval: all authors.

\section{Funding}

This work was financed by FEDER - Fundo Europeu de Desenvolvimento Regional funds through the COMPETE 2020-Operacional Programme for Competitiveness and Internationalization (POCI), Portugal 2020, and by Portuguese funds through FCT-Fundação para a Ciência e a Tecnologia/Ministério da Ciência, Tecnologia e Inovação in the framework of the project "Institute for Research and Innovation in Health Sciences" (POCI01-0145-FEDER-007274). Additional funding by the European Regional Development Fund (ERDF), COMPETE2020 and Portuguese national funds via FCT, under project POCI-01-0145-FEDER-016390: CANCEL STEM and the project POCI-01-0145-FEDER-031438 (PDTC/MED_ONC/31438/2017).

\section{Competing interests}

The authors declare no competing interests.

\section{Additional information \\ Correspondence and requests for materials should be addressed to B.C.}

Reprints and permissions information is available at www.nature.com/reprints.

Publisher's note Springer Nature remains neutral with regard to jurisdictional claims in published maps and institutional affiliations.

(c) (1) Open Access This article is licensed under a Creative Commons Attribution 4.0 International License, which permits use, sharing, adaptation, distribution and reproduction in any medium or format, as long as you give appropriate credit to the original author(s) and the source, provide a link to the Creative Commons licence, and indicate if changes were made. The images or other third party material in this article are included in the article's Creative Commons licence, unless indicated otherwise in a credit line to the material. If material is not included in the article's Creative Commons licence and your intended use is not permitted by statutory regulation or exceeds the permitted use, you will need to obtain permission directly from the copyright holder. To view a copy of this licence, visit http://creativecommons.org/licenses/by/4.0/.

(C) The Author(s) 2021 